quence of acute perichondritis it is ushered in during a few days by symptoms indicating a high degree of inflammation, $e \cdot g$, chill, pronounced fever, pain, swelling and redness of the whole organ. Spontaneous rupture is more apt to occur quickly in this than in the former type, although it may be much delayed after the subsidence of the inflammatory symptoms, leaving again nasal obstruction as the salient symptom.

Case 2 exemplifies the latter type.-Mr. B., aged 26 years; thought he had contracted a severe "cold in the head," the condition commencing with chilly sensations followed by head ache, fever, local sensitiveness and redness extending to the bridge of the nose. These symptoms gradually subsided but were replaced by nasal obstruction and what he now supposed was chronic nasal catarrh. The examination was not made till the third week when a fluctuating tumor was observed to bulge from each side of the septum. Typical pus was evacuated by an incision and the cavity curetted. The destroyed central part of the cartilage ultimately redeveloped without deformity. $\mathrm{He}$ positively denied the least probability of traumatism as a cause.

Diagnosis.-By simple inspection with the head tilted backward, the semicircular tumor may be seen bulging from one or both sides of the septum. Pal. pation by a probe will cause deep pitting or even fluctuation, and aspiration by a hypodermic needle will provide a sample of the contents. It may thus be readily distinguished from polypus for which it is most commonly mistaken by novices, but which occurs but rarely in this situation, and also from syphilitic gumma which commonly develops at just this spot. I have seen one case of gumma in which the central part had liquified before the occurrence of superficial ulceration, in which the diagnosis would have been impossible, had not other syphilitic signs been present.

Prognosis.- If recognized reasonably early and the abscess evacuated, the prognosis is very good. Notwithstanding complete liquefaction of the cartilage itself, if the muco-perichondrium be preserved, snother cartilaginous septum will develop and this usually without deformity. However, if the disease be unrecognized or if the inflammatory action extend to the antero-superior border of the septum, the line of junction of the septum with the lateral cartilage and nasal bones, softening and depression of these which constitute the dorsum of the nose will result. Apart from this "saddle-back" deformity or in conjunction with it, perforation of the septum can also ensue.

Treatment.-When the initial stage is a hematoma cold applications would be suitable. In any event as soon as pus is present a rather free incision should be made low down or one side and the contents expressed. This should be reopened daily with a probe until the cavity has been effaced. It is probably not necessary to curette, wash out or pack the cavity with gauze although one or all of these may seem desirable at times.

\section{FIBROUS NASAL POLYPUS.}

\section{BY D. R. SILVER, M.D.} SIDNEY, OHIO.

As a contribution to our knowledge of nasal polypi, I venture to report this case, which, in one respect at least, may be unique. The growth developed in a boy, 12 years old. He had the characteristic mouthbreathing at all times on account of the complete closure of the posterior nares. On inspection, a tumor the size of a man's thumb could be seen in the naso- pharynx below the line of the soft palate. To the touch it was hard and seemed firmly attached; so firmly, indeed, that to remove it by twisting was out of the question. Its point of attachment could not be made out. Its large size made the passage of anything through either nostril nearly impossible. As a preliminary step, a threaded needle was passed through the lower portion of the growth so that it could be pulled well down and forward, after which it was found possible to pass a small rubber catheter, to which was attached a string, through the right nostril and out of the mouth, as in the operation for closing the posterior nares. By means of the string a heavy silver wire, doubled, was drawn through and slipped over the tumor, fortunately encircling the point of attachment, which could hardly be called a pedicle, since it was found to be nearly as large as any other portion of the neoplasm, and fully threefourths of an inch in diameter. The wire attached to a strong screw cut its way through in an hour.

It proved to be a three-lobed affair, very hard and fibrous, and was drawn out through the mouth by means of the thread which transfixed the growth at its lower end. Its dimensions were $2 \frac{3}{4}$ by $1 \frac{1}{3}$ by $\frac{3}{4}$ inches. The pain produced by the constricting wire was considerable, but there was no hemorrhage to occasion alarm. Notwithstanding the fact that the point of attachment was well cauterized a large mucous polypus sprang up in a few weeks. This was removed by twisting and did not return. The specialist trained in the manipulations of rhinologic work may not find the removal of nasal and post-nasal polypi very difficult. But when these growths are very large or very small the general practitioner finds their management quite perplexing. For this reason the subjects of nasal polypi furnish a large contingent of the army of victims who fall an easy prey to the catarrh "specialist." Nostrums, whose only recommendation is their nastiness, are bought and used for years by persons whose rather fashionable complaint could be removed by a twist of the forceps.

\section{HEMORRHAGE FROM THE LARYNX, FOL- LOWING AN ATTACK OF MEASLES, IN AN ADULT.}

Read before the Section of Otology and Laryngology of the College of Physicians, Philadelphia, Pa.. March 3, 1896.

BY FRANK WOODBURY, M.D.

Instructor in Laryngology in the Philadelphia Polyclinic; Attending Physician to the Medico-Chirurgical Hospital, etc. PHILA DELPHIA, PA.

On January 24, Martin S., 27 years of age, born in Ireland, a laborer by occupation, presented himself at the laryngologic dispensary of the Polyclinie Hospital with an acute inflammation of the fauces and pharynx and with almost extinguished voice, requiring considerable effort in speaking. He gave the following history: About nine weeks previous, or about the first of December, four sailors were admitted to the Naval Hospital suffering with measles. Three cases developed subsequently among the employes of the institution, where he was engaged as a fireman, and all the cases recovered. The patient was taken sick January 3, or about two weeks after the first patients were convalescent, and had been allowed to go around the grounds. He said that he had fever, eruption, conjunctivitis, cough, and subsequently there was much desquamation, especially from his face 